Table 1 - Gender, age, medications, HAM-A and BPRS scores

\begin{tabular}{|c|c|c|c|c|c|c|c|c|c|c|}
\hline \multirow{2}{*}{ Number } & \multirow{2}{*}{ Gender } & \multirow{2}{*}{$\begin{array}{c}\text { Age } \\
\text { (years) }\end{array}$} & \multirow{2}{*}{$\begin{array}{l}\text { Clozapine daily } \\
\text { dose }\end{array}$} & \multirow{2}{*}{$\begin{array}{l}\text { Milnacipran daily } \\
\text { dose }\end{array}$} & \multicolumn{3}{|c|}{ HAM-A } & \multicolumn{3}{|c|}{ BPRS } \\
\hline & & & & & TO & T4 & T8 & TO & T4 & T8 \\
\hline 1 & Male & 40 & $700 \mathrm{mg}$ & $100 \mathrm{mg}$ & 20 & 13 & 9 & 52 & 42 & 28 \\
\hline 2 & Male & 33 & $900 \mathrm{mg}$ & $150 \mathrm{mg}$ & 33 & 23 & 28 & 78 & 75 & 83 \\
\hline 3 & Male & 30 & $700 \mathrm{mg}$ & $100 \mathrm{mg}$ & 29 & 22 & 25 & 45 & 41 & 46 \\
\hline 4 & Male & 42 & $600 \mathrm{mg}$ & $150 \mathrm{mg}$ & 36 & 34 & 34 & 93 & 91 & 91 \\
\hline 5 & Female & 36 & $700 \mathrm{mg}$ & $150 \mathrm{mg}$ & 38 & 37 & 34 & 80 & 83 & 78 \\
\hline 6 & Female & 29 & $600 \mathrm{mg}$ & $150 \mathrm{mg}$ & 36 & 32 & 38 & 75 & 67 & 70 \\
\hline
\end{tabular}

effects of milnacipran on anxiety, it has been reported to be efficacious in the treatment of depression in patients with schizophrenia spectrum disorders. ${ }^{5}$

Six outpatients (4 males, 2 females) diagnosed with schizophrenia according to the DSM-IV and intense anxiety symptoms, although not meeting the criteria for any DSM-IV anxiety disorder, were studied in order to determine the efficacy of milnacipran in reducing anxiety symptoms in this population. All patients were under treatment with clozapine for refractory schizophrenia.

The mean age of the subjects was $35 \pm 5$ years. The mean daily doses of clozapine and milnacipran were $700 \mathrm{mg}$ and $100 \mathrm{mg}$, respectively. All subjects were submitted to blood workups as recommended for clozapine-treated patients. No hematologic abnormalities were found in this group.

The efficacy of milnacipran in reducing anxiety symptoms, as characterized according to the Hamilton Anxiety Scale (HAMA), which was applied before treatment (TO), after 4 weeks of treatment (T4) and after 8 weeks of treatment (T8). The Brief Psychiatric Rating Scale (BPRS) was also applied at T0, T4 and T8 in order to evaluate possible worsening of schizophrenia symptoms due to treatment with the antidepressant. Comparisons between groups were made using paired Student's $t$-tests. Table 1 shows the demographic data for the patients treated with milnacipran, as well as variations between TO HAM-A and BPRS scores and those obtained at T4 and T8. The mean HAMA scores decreased significantly $(p=0.014)$ from T0 $(32 \pm 7)$ to T4 (27 \pm 9$)$, remaining stable between $\mathrm{T} 4$ and T8 $(28 \pm 10 ; p=0.523)$. From T0 to T8, a trend toward decreasing HAM-A was found ( $p=0.069)$. The mean BPRS score remained virtually unchanged $(p=0.087)$ from TO $(71 \pm 18)$ to T4 $(67 \pm 21)$, from T4 to T8 $(66 \pm 24)(p=0.884)$ and from TO to T8 ( $p=0.326)$. No patient reported adverse effects related to the treatment.

Clearly, the small size of our sample precludes generalization, and studies involving larger samples are needed to confirm these findings. However, our results suggest that milnacipran might be effective in reducing anxiety symptoms in the fourth week of use, and this effect might be maintained until the eighth week of use. In addition, the tolerability profile and the low potential for drug interactions presented by milnacipran could make it a very useful alternative for treating anxiety in patients using clozapine, without the risk of drug interactions and worsening psychotic symptoms.

Clarissa Severino Gama, Vanessa Cassina Zanatto, Felipe Picon, Maria Inês Lobato, Paulo Silva Belmonte-de-Abreu Department of Psychiatry, Hospital de Clínicas de Porto Alegre (HCPA), Universidade Federal do Rio Grande do Sul (UFRGS), Porto Alegre (RS), Brazil
References

1. Cosoff SJ, Hafner RJ. The prevalence of comorbid anxiety in schizophrenia, schizoaffective disorder and bipolar disorder. Aust N Z J Psychiatry. 1998;32(1)67-72.

2. Pallanti S, Quercioli L, Hollander E. Social anxiety in outpatients with schizophrenia: a relevant cause of disability. Am J Psychiatry. 2004;161(1):53-8.

3. Wetherell JL, Palmer BW, Thorp SR, Patterson TL, Golshan S, Jeste DV. Anxiety symptoms and quality of life in middle-aged and older outpatients with schizophrenia and schizoaffective disorder. J Clin Psychiatr. 2003;64(12):1476-82.

4. Braga RJ, Mendlowicz MV, Marrocos RP, Figueira IL. Anxiety disorders in outpatients with schizophrenia: prevalence and impact on the subjective quality of life. J Psychiatr Res. 2005;39(4):409-14

5. Nakanishi S, Kunugi H, Takahashi T. Efficacy of milnacipran for depressive symptoms in schizophrenia spectrum disorders. Psychiatr Clin Neurosci. 2004;58(2):226-7.

\section{Autopercepção da comunicação oral no tratamento de adolescentes usuários de drogas}

\section{Oral communication self-perception in drugis}

users adolescents

\section{Sr. Editor,}

É por meio da comunicação, independente de sua modalidade, que o ser humano expressa conhecimentos, emoções, intenções, desejos e insatisfações. A comunicação oral, a mais utilizada nas relações pessoais, é efetiva quando uma série de habilidades é estabelecida entre locutor e interlocutor: saber ouvir, compartilhar um mesmo código lingüístico e ter discernimento sobre o que, onde e como falar, entre outros.

Apesar de o uso de drogas alterar a comunicação oral e a relação do usuário com o seu meio, raros são os estudos que enfocam essa questão. ${ }^{1-3} \mathrm{O}$ usuário parece não processar o que ouve e tampouco tem consciência de como e o que fala, talvez porque seus interesses centram-se na droga. As intenções implicadas em sua comunicação passam a ter caráter duvidoso e as vontades são, na maioria das vezes, ignoradas. O usuário, por viver em um círculo social restrito, passa a se comunicar de forma peculiar, similar à de outros usuários e essa comunicação é relativamente efetiva, apenas e somente nesse meio.

Com o intuito de pesquisar como se processa a comunicação oral de adolescentes usuários de drogas e os diversos sentidos da mesma, 31 adolescentes de ambos os sexos, do Ambulatório de Adolescentes e Drogas do Serviço de Infância e Adolescência do Instituto de Psiquiatria do Hospital das Clínicas da Faculdade de Medicina da Universidade de São Paulo (CAPPesq 636/03) foram encaminhados por seus psiquiatras ao fonoaudiólogo, que solicitou aos mesmos que tentassem narrar fatos ocorridos na infância e os mais recentes relacionados do cotidiano. As narra- 
tivas foram gravadas e imediatamente ouvidas pelos adolescentes para que julgassem sobre sua própria comunicação.

Este procedimento permitiu verificar que grande parte dos adolescentes estranhou a forma pela qual estavam se comunicando. Esta descoberta propiciou a abertura de um espaço de comunicação que, para alguns, se expandiu para novas conversas e que, por não terem caráter obrigatório de consulta, permitiriam que o adolescente entrasse em contato com a própria comunicação e iniciasse um processo de conscientização sobre o sentido e a forma da mesma.

Nas conversas, a relação do adolescente com a droga foi um dos temas e, como conseqüência, o fonoaudiólogo propôs tentativas de abstinência da mesma, que poderia ou não ter resultados. Essa solicitação foi possível porque se estabeleceu um canal efetivo de comunicação iniciado pelo próprio adolescente, que se sentiu seguro para falar sobre o uso e, muitas vezes, sobre a implicação deste em sua vida, com um profissional voltado para a comunicação humana.

Para melhor análise do procedimento utilizado, foram consultados prontuários de 31 pacientes com semelhantes diagnósticos de uso de drogas, mas que ingressaram no tratamento psiquiátrico antes do início da abordagem fonoaudiológica, permitindo obter informações sobre o comportamento de ambos os grupos quanto ao tratamento psiquiátrico.

Comparando-se os dois grupos, os resultados indicaram que os adolescentes que haviam sido consultados pelo fonoaudiólogo permaneceram 30 semanas em tratamento psiquiátrico, enquanto aqueles que não foram atendidos por esse profissional permaneceram 13 semanas ( $t$-student, $p=0,023$ ). Outro dado importante mostrou que os adolescentes usuários de maconha que tiveram contato com o fonoaudiólogo permaneceram 17 semanas abstinentes, enquanto os que não foram atendidos por este profissional, 6 semanas ( $p=0,01$ ).

Este estudo mostrou que a autopercepção ou o processo de consciência da comunicação dos adolescentes usuários de drogas, assim como o espaço individual e aberto com o fonoaudiólogo propiciou, ao menos, a busca pelo autoconhecimento e a reflexão sobre o próprio comportamento, resultando em maior tempo de abstinência. Isto se deu na relação ouvir-falar-interagir, que contribuiu para uma mudança no padrão do uso de drogas.

Christian César Cândido de Oliveira Ambulatório de Adolescentes e

Drogas, Serviço de Psiquiatria da Infância e Adolescência (SEPIA), Hospital das Clínicas, Faculdade de Medicina, Universidade de São Paulo (USP), São Paulo (SP), Brasil

Claudia Ines Scheuer Programa de Pós-graduação em Ciências da Reabilitação, Faculdade de Medicina, Universidade de São Paulo (USP),

São Paulo (SP), Brasil

Sandra Scivoletto

Grupo Interdisciplinar de Estudos de Álcool e Drogas, Instituto de Psiquiatria, Hospital das Clínicas, Faculdade de Medicina, Universidade de São Paulo (USP),

São Paulo (SP), Brasil

Ambulatório de Adolescentes e Drogas, Serviço de Psiquiatria da Infância e Adolescência (SEPIA), Hospital das Clínicas, Faculdade de Medicina, Universidade de São Paulo (USP), São Paulo (SP), Brasil

Programa de Pós-Graduação em Psiquiatria e Fisiopatologia Experimental, Faculdade de Medicina, Universidade de São Paulo (USP), São Paulo (SP), Brasil
Financiamento: Inexistente

Conflito de interesses: Inexistente

Referências

1. Oliveira CCC, Scivoletto S. Percepción de los efectos del alcohol y otras drogas en el habla y el lenguaje en consumidores habituales en edad adulta. Revista de Logopedia, Foniatria y Audiologia. 2004;24(3): 126-31.

2. Solowij N, Stephens RS, Roffman RA, Babor T, Kadden R, Miller M, Christiansen K, Mcree B, Vendetti J. Marijuana Treatment Project Research Group. Cognitive functioning of long heavy cannabis users seeking treatment. JAMA. 2002;287(9):1123-31.

3. Messinis L, Kyprianidou A, Malefaki S, Papathanasopoulos P. Neuropsychological deficits in long-term frequent cannabis users. Neurology. 2006;66(5):737-9.

\section{Alopecia secundária ao uso de inibidor seletivo da recaptação de serotonina: relato de dois casos}

\author{
Alopecia associated with the use of serotonin \\ selective reuptake inhibitors: two case reports
}

\section{Sr. Editor,}

A perda de cabelos pode ser um efeito adverso de inúmeros psicofármacos, com alguns relatos envolvendo o uso de antidepressivos. ${ }^{1-2}$ A seguir, descrevemos dois casos de pacientes que desenvolveram alopecia secundariamente ao uso de um inibidor seletivo da recaptação de serotonina (ISRS).

Caso 1 - LAFR, 47 anos, sexo feminino, leucoderma, diagnosticada com transtorno de ansiedade generalizada. Iniciou fluoxetina $10 \mathrm{mg} / \mathrm{d}$ com aumento progressivo para $20 \mathrm{mg} / \mathrm{d}$. Após aproximadamente cinco semanas, queixou-se de "cabelos fracos", com sua escova ficando cheia de cabelos quando se penteava, fato que nunca ocorrera. No terceiro mês de tratamento, apresentava importante redução dos sintomas ansiosos, porém com importante perda capilar do couro cabeludo. Após substituição da medicação pela paroxetina $(20 \mathrm{mg} / \mathrm{d})$, houve recuperação gradual e completa dos cabelos em um período de dois meses, sem reaparecimento dos sintomas ansiosos.

Caso 2 - DDS, 25 anos, sexo masculino, leucodermo, primo da primeira paciente, recebeu o diagnóstico de bulimia nervosa e episódio depressivo leve. Segundo o paciente, os episódios de hiperfagia seguida de vômitos auto-induzidos tiveram início há, aproximadamente, dois anos, após ter passado por período de "estresse e ansiedade". Foi iniciado citalopram $20 \mathrm{mg} / \mathrm{d}$ e psicoterapia. Após cinco meses, mesmo sem melhora da sintomatologia psiquiátrica, interrompeu uso do fármaco devido à expressiva redução capilar do couro cabeludo, axilas e tórax. Manteve o tratamento psicoterápico, mas recusou-se a utilizar outros fármacos com medo de ficar "careca". Após cinco meses, havia recuperado totalmente os pêlos das regiões acometidas, sem redução dos sintomas psiquiátricos.

Ambos os pacientes passaram por avaliação clínica (sem alterações na propedêutica hepática, renal, hematológica e da tireóide) e dermatológica completa, nunca apresentaram quei- 